\title{
Blood pressure variability and closed-loop baroreflex assessment in adolescent chronic fatigue syndrome during supine rest and orthostatic stress
}

\author{
Vegard Bruun Wyller • Riccardo Barbieri • \\ J. Philip Saul
}

Accepted: 20 September 2010/Published online: 2 October 2010

(C) The Author(s) 2010. This article is published with open access at Springerlink.com

\begin{abstract}
Hemodynamic abnormalities have been documented in the chronic fatigue syndrome (CFS), indicating functional disturbances of the autonomic nervous system responsible for cardiovascular regulation. The aim of this study was to explore blood pressure variability and closedloop baroreflex function at rest and during mild orthostatic stress in adolescents with CFS. We included a consecutive sample of 14 adolescents 12-18 years old with CFS diagnosed according to a thorough and standardized set of investigations and 56 healthy control subjects of equal sex and age distribution. Heart rate and blood pressure were recorded continuously and non-invasively during supine rest and during lower body negative pressure (LBNP) of $-20 \mathrm{mmHg}$ to simulate mild orthostatic stress. Indices of blood pressure variability and baroreflex function ( $\alpha$-gain) were computed from monovariate and bivariate spectra in the low-frequency (LF) band $(0.04-0.15 \mathrm{~Hz})$ and the highfrequency (HF) band $(0.15-0.50 \mathrm{~Hz})$, using an autoregressive algorithm. Variability of systolic blood pressure in the
\end{abstract}

Communicated by Dag Linnarsson.

V. B. Wyller $(\square)$

Department of Pediatrics,

Rikshospitalet University Hospital,

0027 Oslo, Norway

e-mail: brwylle@online.no

R. Barbieri

Department of Anesthesia and Critical Care,

Massachusetts General Hospital,

Harvard Medical School, Boston, USA

J. P. Saul

Department of Pediatrics,

Medical University of South Carolina,

Charleston, USA
HF range was lower among CFS patients as compared to controls both at rest and during LBNP. During LBNP, compared to controls, $\alpha$-gain HF decreased more, and $\alpha$-gain $\mathrm{LF}$ and the ratio of $\alpha$-gain LF/ $\alpha$-gain HF increased more in CFS patients, all suggesting greater shift from parasympathetic to sympathetic baroreflex control. CFS in adolescents is characterized by reduced systolic blood pressure variability and a sympathetic predominance of baroreflex heart rate control during orthostatic stress. These findings may have implications for the pathophysiology of CFS in adolescents.

Keywords Blood pressure variability - Baroreflex sensitivity $\cdot$ Chronic fatigue syndrome $\cdot$ Autonomic nervous system $\cdot$ Cardiovascular regulation

\section{Introduction}

The chronic fatigue syndrome (CFS) is a disabling disease, mainly affecting adolescents and young adults (Natelson 2001); recently, the prevalence among $8-17$ years olds was reported as high as $1.3 \%$ (Farmer et al. 2004). Certain infections (such as mononucleosis) and dramatic life events are considered important precipitating factors (Natelson 2001). The condition usually last for years, but spontaneous improvement is common, particularly among adolescents.

The CFS pathophysiology is unknown, but recent evidence suggests that abnormalities of cardiovascular regulation may play an important role. Various forms of orthostatic intolerance have been demonstrated both in adult (Bou-Holaigah et al. 1995) and pediatric (Galland et al. 2008; Rowe et al. 1995; Rowe and Calkins 1998; Wyller et al. 2007a, 2008b) patients, as well as abnormalities in cerebral (Tanaka et al. 2002), muscle (McCully et al. 2004) 
and skin (Spence et al. 2000; Wyller et al. 2007b) hemodynamics. Taken together, these observations indicate that CFS is characterized by functional disturbances of the autonomic nervous system affecting cardiovascular regulation.

One direction of investigation has applied variability analyses of heart rate and blood pressure during orthostatic challenge to explore in more detail the mechanisms of autonomic cardiovascular control in CFS. However, CFS studies based on such methodology have yielded conflicting results (Boneva et al. 2007; Duprez et al. 1998; Stewart 2000; Yataco et al. 1997; Yoshiuchi et al. 2004). Two reasons may account for this. First, most studies use an ordinary head-up tilt-test for the orthostatic challenge. However, in an experimental setting, the test may not be ideal due to the varying effects of the muscle venous pump among different participants in some experimental protocols and the high rate of false positives in adolescents (De Jong-De Vos van Steenwijk et al. 1995). Second, the mathematical algorithm used for variability analyses may not be optimal. For instance, when estimating baroreflex sensitivity, most techniques fail to account for the feedforward effect (i.e. the mechanical influence) of heart rate on blood pressure (Barbieri et al. 1996).

The technique of lower body negative pressure (LBNP) is generally considered to mimic orthostasis effectively. Compared to most upright tilt protocols, it provides a more accurate tool for studies of cardiovascular adjustments during orthostatic stress because the subjects do not move, and the effect of the muscle venous pump is eliminated (Stevens and Lamb 1965). Previously, we have reported evidence of altered heart rate variability in CFS patients during LBNP, indicating a predominance of sympathetic heart rate control during mild orthostatic stress (Wyller et al. 2008a). However, the autonomic neurons controlling heart rate are continuously influenced by different sources, including baroreceptor afferents, respiration and central command (Saul 1990). Thus, the aim of the present study was to assess blood pressure variability and one of the factors that influence both RR and blood pressure variability, the arterial baroreflex, both at rest and during LBNP. We hypothesized that indices of blood pressure variability and baroreflex function would differ between CFS patients and controls, indicating a predominance of sympathetic cardiovascular control in the former.

\section{Methods}

\section{Subjects}

CFS patients $12-18$ years old were consecutively recruited from the outpatient clinic at the Department of Pediatrics, Rikshospitalet University Hospital, Oslo, Norway, serving as a national referral center for children and adolescents with unexplained chronic fatigue. Based upon our previous experiences, we assumed that a total number of approximately 15 CFS patient would be sufficient (Wyller et al. 2008a, b) Other disease states that might explain their present symptoms, such as autoimmune, endocrine, neurologic or psychiatric disorders, were ruled out by a thorough and standardized set of investigations. Different case definitions of CFS exist. This study used a slight modification of the definition from the Centers for Disease Control and Prevention (CDC). The main criterion of at least 6 months of chronic or relapsing fatigue, severely affecting daily activities (Fukuda et al. 1994), was required in this study. However, the CDC definition also requires patients having at least four of eight specific accompanying symptoms. Since the validity of this last demand has been questioned (Cho et al. 2006), particularly in the pediatric population (Franklin 1998), accompanying symptoms were not required in this study.

Healthy controls of 12-18 years old volunteered from local schools. In line with our previous experimental studies (Wyller et al. 2008a, b), we sought a 1:4 relation between patients and controls in order to increase the statistical power. Through communication with the responsible teachers, a recruiting process was established that assured an equal distribution of age and sex among the two groups. Subjects having a chronic disease (such as allergy) or using any medications on a regular basis (including contraceptive pills) were excluded.

One week prior to the experiments, all participants were instructed not to drink beverages containing alcohol or caffeine, not to take any drugs and not to use tobacco products. They were instructed to fast overnight the day prior to the experiments.

Written, informed consent was obtained from all participants and their parents. The study was approved by the Regional committee for ethics in medical research. The RR variability data from these same subjects were reported previously (Wyller et al. 2008a).

Lower body negative pressure (LBNP) with handgrip

Experiments started at 11 a.m. The participants had been offered a light, standardized meal (1-2 pieces of bread, 1 glass of juice) $2 \mathrm{~h}$ before, but were otherwise not allowed to eat or drink. They lay supine with their lower body in a plastic chamber, in which air could be evacuated very rapidly, thus reaching a pre-defined negative pressure within milliseconds (Hisdal et al. 2003). In order to prevent air leak, rubber devices were used to make a tight seal around the subjects' waist. They were lightly dressed; the ambient temperature was kept between 23 and $26^{\circ} \mathrm{C}$, and time of approximately $30 \mathrm{~min}$ was used for acclimatization 
prior to experiments. They were familiarized with the test situations in two pilot experiments.

Time of 5 min was used for baseline registration of cardiovascular variables. Then LBNP of $-20 \mathrm{mmHg}$ was applied. After 6 min of LBNP, the subjects were asked to perform left-sided handgrip for 1 min with $30 \%$ of maximal voluntary contraction force. This procedure was repeated once after a 1-min resting interval. One minute after termination of the second handgrip, LBNP was turned off. The cardiovascular adjustments during combined LBNP and handgrip have been reported elsewhere (Wyller et al. 2008b).

The procedure was performed twice with a 5-min rest period between applications of LBNP. However, in two subjects (one patient and one control), only one complete run of LBNP and handgrip was performed due to dizziness or other unpleasant experience. Two additional recordings were excluded from the analyses due to low technical quality.

Instantaneous heart rate (HR) was obtained from the $\mathrm{R}-\mathrm{R}$ interval (RRI) of the ECG. Photoplethysmography on the right middle finger was used to obtain a non-invasive, continuous recording of arterial blood pressure (2300 Finapres, Ohmeda, Madison, WI, USA). This method correlates satisfactorily with invasive pressure measurements (Parati et al. 1989) and has also been validated in adolescents and children (Seifer and Kenny 2001). In addition, non-invasive techniques were used for continuous recording of aortic blood flow (bidirectional ultrasound Doppler velocimeter located at the suprasternal notch), brachial artery blood flow (bidirectional ultrasound Doppler velocimeter located at the elbow) and acral skin blood flow (laser-Doppler probe located at the right index finger). These measurements are not reported in this paper. All recorded signals, including the pressure in the LBNP chamber, were on-line transferred to a recording computer running a program for real-time data acquisition (developed by Morten Eriksen, Department of Physiology, University of Oslo, Norway).

RRI and BP monovariate spectral analysis

Beat-to-beat series of RRI and maximum (systolic: SBP) and minimum (diastolic: DBP) interbeat arterial blood pressure (ABP) values were converted to $3-\mathrm{Hz}$ time series. For each experimental run, segments of $120 \mathrm{~s}$ length were selected, respectively, prior to LBNP and during LBNP prior to the handgrip periods, and subjected to spectral analyses using an autoregressive algorithm (Barbieri et al. 2001). Spectral components were decomposed, and power densities were computed in the low-frequency band $(0.04-0.15 \mathrm{~Hz})$ and the high-frequency band $(0.15-0.5 \mathrm{~Hz})$. For subjects with two experimental recordings, the arithmetical mean for each variable was computed from corresponding experimental epochs.
Spectral closed-loop analysis

The 3-Hz time series was also analyzed by a bivariate AR Yule-Walker algorithm (Barbieri et al. 2001), where the bivariate autoregressive model of order $p$ is described by the following vector equation:

$Y[n]=\sum_{k=1}^{p} A[k] Y[n-k]+W[n]$.

When applied to the interactions between SBP (or DBP) and RRI, the matrices are defined as:

$$
\begin{gathered}
\mathbf{A}[k]=\left[\begin{array}{ll}
a_{11}[k] & a_{12}[k] \\
a_{21}[k] & a_{22}[k]
\end{array}\right], \quad \mathbf{Y}[n]=\left[\begin{array}{c}
R R I[n] \\
A B P[n]
\end{array}\right], \\
\mathbf{W}[n]=\left[\begin{array}{l}
w_{R R I}[n] \\
w_{A B P}[n]
\end{array}\right] .
\end{gathered}
$$

The $p$ matrices of coefficients $A(k)$ were calculated by solving the extended Yule-Walker equations. The order $p$ of the model was fixed at 16 throughout the analysis. This was done in order to guarantee a homogeneous, high-order across-all segment. Of note, this order was generally higher than the minimum order required by the Akaike criterion.

The bivariate coefficients were used to simultaneously analyze the gains and phases for the following transfer functions:

$G_{\mathrm{ABP} \rightarrow \mathrm{RR}}(f)=\frac{A_{12}(f)}{1-A_{11}(f)}$,

the heart rate baroreflex feedback (SBP $\rightarrow$ RRI and $\mathrm{DBP} \rightarrow \mathrm{RRI}$, or alpha), and

$G_{\mathrm{RR} \rightarrow \mathrm{ABP}}(f)=\frac{A_{21}(f)}{1-A_{22}(f)}$,

the feedforward from RRI to ABP (RRI $\rightarrow$ SBP and $\mathrm{RRI} \rightarrow \mathrm{DBP}$, or beta), where

$A_{i j}(f)=\sum_{k=1}^{p} a_{i j}[k] e^{-j 2 \pi f k}$.

The feedback SBP $\rightarrow$ RRI gain was taken as a measure of the sensitivity of closed-loop baroreflex modulation of RRI, while the feedforward RRI $\rightarrow$ DBP gain was taken as the measure of the sensitivity of the closed-loop mechanical coupling between RRI and DBP fluctuations. Phase relationships were also assessed to investigate the delays in the SBP-RRI and RRI-DBP interactions.

Gain and phase values were extracted in the low-frequency (LF) band $(0.04-0.15 \mathrm{~Hz})$ and the high-frequency (HF) band $(0.15-0.5 \mathrm{~Hz})$, anywhere the coherence between RRI and SBP signals reached its maximum inside each frequency band (Fig. 1). As previously suggested, the $\alpha$-gain for baroreflex feedback was characterized between SBP and RR, and the $\beta$-gain feedforward was characterized 
Baseline
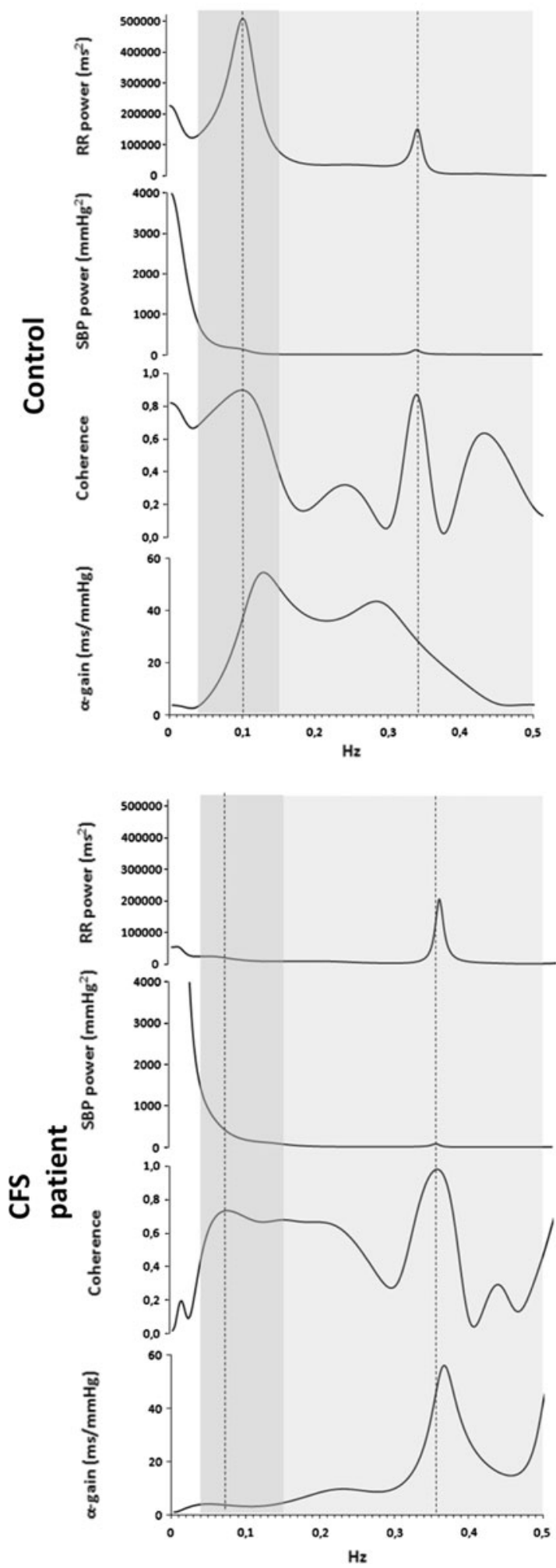

LBNP
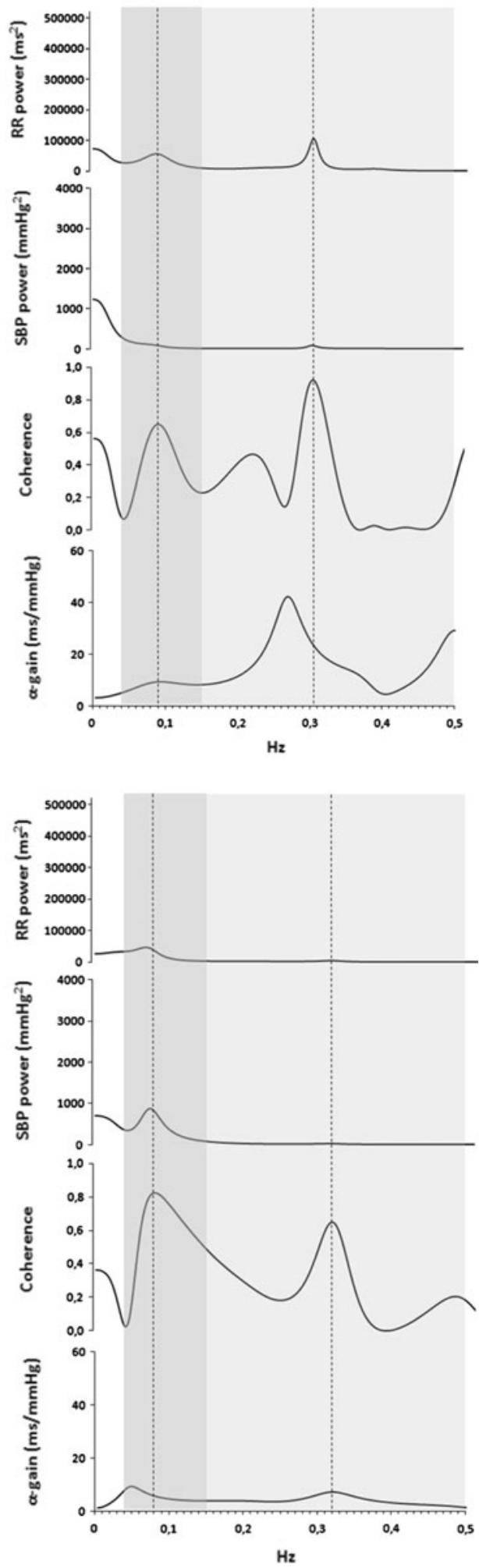

Fig. 1 Individual univariate and bivariate spectra from one healthy control (upper panels) and one CFS patient (lower panels) at baseline (left) and during LBNP (right). Dark shadowed areas indicate the low-frequency range $(0.04-0.15 \mathrm{~Hz})$; light shadowed areas indicate the high-frequency range $(0.1-0.50 \mathrm{~Hz})$. Vertical dotted lines mark the point of maximal coherence within each frequency band. Of note, in the healthy control, LBNP results in a reduction of $\alpha$-gain in both frequency bands, whereas in the CFS patient, LBNP is associated with a decrease of $\mathrm{HF} \alpha$-gain and an increase in LF $\alpha$-gain 
between RR and DBP because of the direct effects of changes in the RR interval on DBP (Barbieri et al. 2001). For subjects with two experimental recordings, the arithmetical mean for each variable was computed from corresponding experimental epochs.

\section{Statistical analyses}

Statistical analyses were carried out using SPSS statistical software. Based upon inspection of plots, most variables were appraised not to follow a normal distribution. Thus, results are expressed as median with non-parametric $95 \%$ confidence intervals. The non-parametric Wilcoxon-MannWhitney's test (two-sided) was used to explore the differences between the two groups. Since the research questions did not concern within group differences, statistical tests for repeated measurements were not applied. A $p$ value of $\leq 0.05$ was considered statistically significant. In order to reduce the methodological problem of multiple comparisons, statistical tests were only performed for the cardiovascular variables considered to be most relevant to our hypothesis.

\section{Results}

A total of 14 CFS patients and 56 healthy controls were included in the study (Table 1). The two groups were comparable regarding sex, age, weight and height. All were of Caucasian ethnicity, except one control.

Table 1 Subject characteristics

\begin{tabular}{lcc}
\hline & $\begin{array}{c}\text { Control }(n=56) \\
\text { Mean (range) }\end{array}$ & $\begin{array}{l}\text { CFS }(n=14) \\
\text { Mean (range) }\end{array}$ \\
\hline Number of patients & 56 & 14 \\
Female gender, $n(\%)$ & $33(58.9)$ & $9(64.3)$ \\
Age (years) & $15.6(13-18)$ & $15.2(12-18)$ \\
Weight $(\mathrm{kg})$ & $61.6(44-99)$ & $59.5(43-92)$ \\
Height $(\mathrm{cm})$ & $171.5(149-195)$ & $172.2(160-192)$ \\
Body surface area (m $\left.{ }^{2}\right)$ & $1.7(1.4-2.2)$ & $1.7(1.4-2.2)$ \\
Duration of fatigue (months) & & $31.3(6-60)$ \\
\hline
\end{tabular}

Mean duration of fatigue among the patients was 31 months. Their functional impairments were severe. They were physically inactive, did not participate in leisure activities and had a high level of school absenteeism. However, no one was permanently bedridden. HR and DBP were increased, and RRI was decreased both at rest and during LBNP compared to controls (Table 2).

Results from the univariate spectral analyses of blood pressure signals are shown in Tables 3 and 4 and Fig. 1. For SBP, $\mathrm{HF}_{\mathrm{abs}}$ at baseline was significantly lower among CFS patients than controls. These differences persisted during LBNP. DBP variability indices did not differ among the two groups.

Results from bivariate spectral analyses are shown in Tables 5 and 6 and Fig. 1. At rest, the $\alpha$-phase HF for the SBP-RR spectrum was more negative among CFS patients than controls. Otherwise, no significant differences were identified among the two groups at rest. However, during LBNP, $\alpha$-gain HF tended to decrease more, and $\alpha$-gain LF tended to increase more among CFS patients than controls, leading to a marked increase in the ratio of $\alpha$-gain LF/ $\alpha$-gain HF in the CFS-patients which was not present in the controls. Also during LBNP, $\alpha$-phase LF was less negative in CFS patients than in controls. For feedforward $\beta$-gain, no significant differences were seen between CFS patients and controls for any of the parameters measured. For all computed bivariate spectra, median coherence values were acceptable in the range of $0.66-0.83$.

\section{Discussion}

The most important finding of this study is that the ratio of LF:HF gain for baroreflex feedback increases markedly in CFS patients in response to orthostatic stress, compared to virtually no change in normal controls. The significant increase in the gain ratio was due to a combination of increased LF gain and decreased HF gain in the CFS group, while the controls only had a reduction in HF gain. These findings indicate that mild orthostatic stress leads to a small reduction of parasympathetically mediated heart rate control in both CFS patients and controls (decreased HF gain),

Table 2 Standard hemodynamic variables at baseline and during LBNP - $20 \mathrm{mmHg}$ : median (confidence interval)

\begin{tabular}{|c|c|c|c|c|}
\hline & \multicolumn{2}{|l|}{ Baseline } & \multicolumn{2}{|l|}{ LBNP } \\
\hline & Control $(n=56)$ & $\mathrm{CFS}(n=14)$ & Control $(n=56)$ & $\mathrm{CFS}(n=14)$ \\
\hline Heart rate (beats/min) & $65.1(62.0-67.6)$ & $71.9(66.1-81.5)$ & $70.8(67.6-75.7)$ & $86.2(80.6-93.0)$ \\
\hline RR-interval (ms) & $921(887-968)$ & $834(736-908)$ & 847 (793-887) & $696(645-744)$ \\
\hline Systolic blood pressure $(\mathrm{mmHg})$ & $118.6(113.5-123.9)$ & $115.6(110.5-130.3)$ & $117.4(112.9-122.1)$ & $118.2(110.7-133.1)$ \\
\hline Diastolic blood pressure $(\mathrm{mmHg})$ & $63.8(62.1-66.0)$ & $69.4(65.5-74.9)$ & $66.3(64.1-67.9)$ & $73.8(68.7-79.5)$ \\
\hline
\end{tabular}



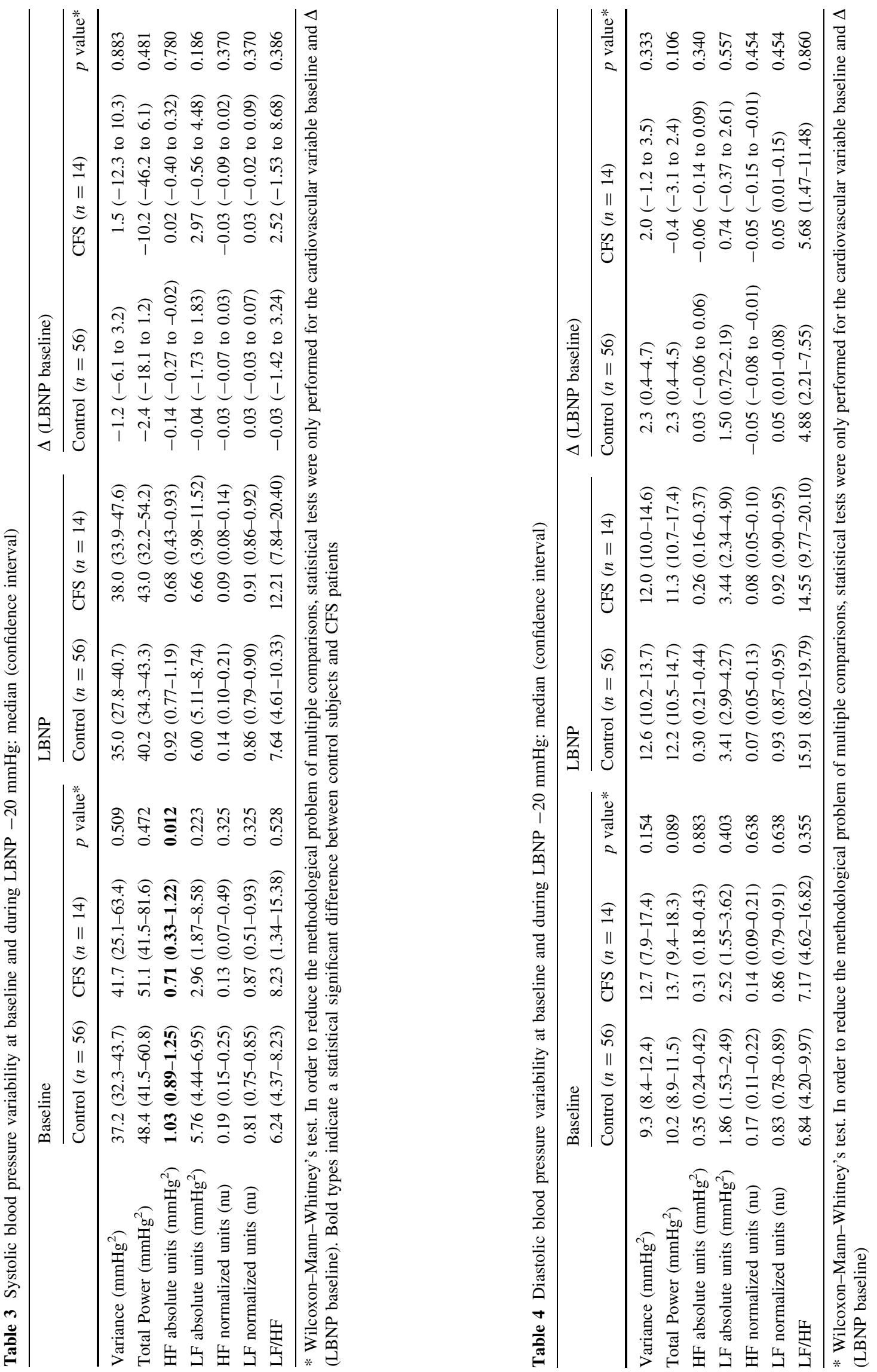

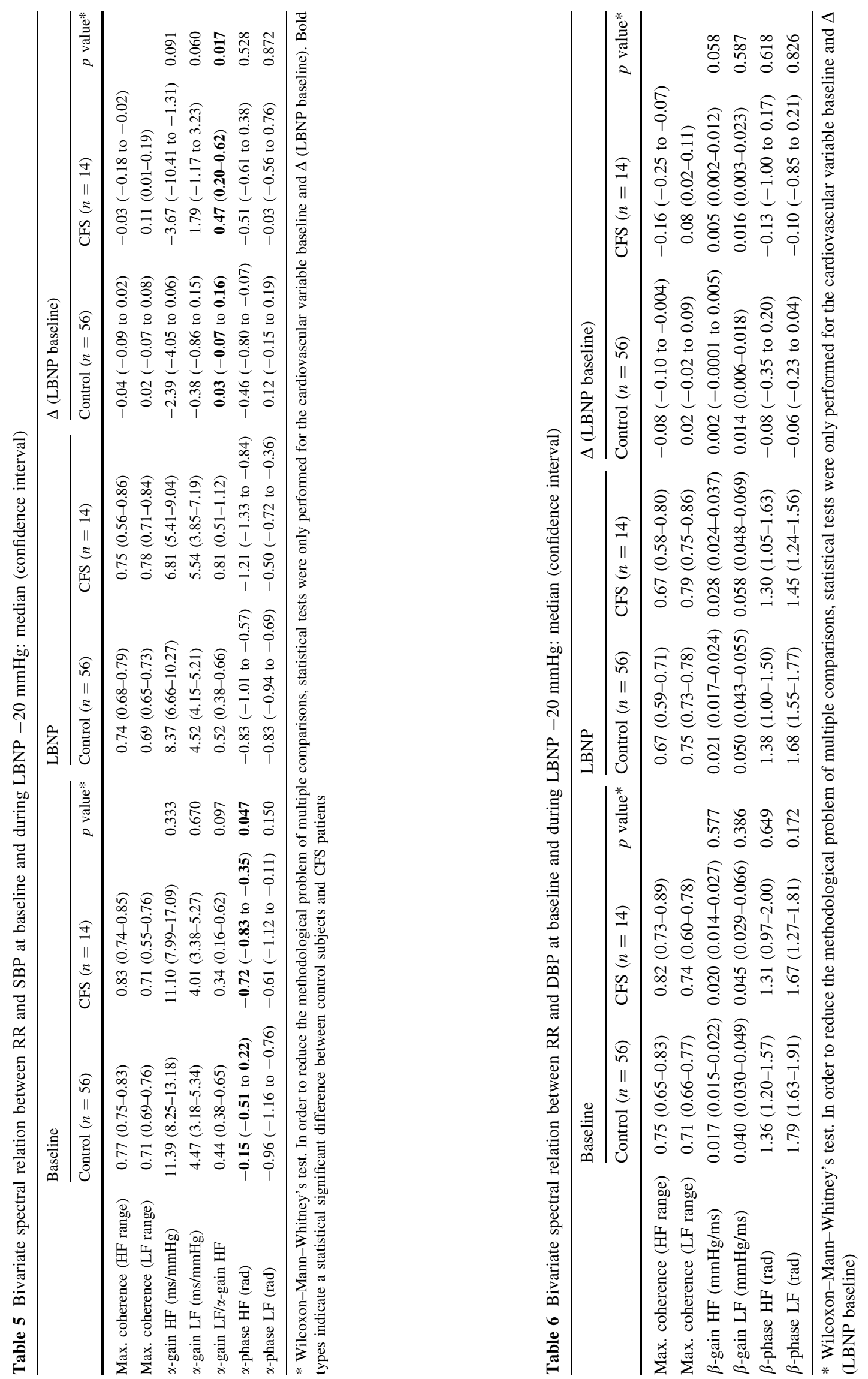
but a significant increase in sympathetically mediated baroreflex gain in CFS patients (increased LF gain) which is not observed in controls.

In addition, reduced HF SBP variability was observed in CFS patients compared to controls, possibly reflecting a reduced effect of respiration on RR or SBP in CFS patients.

\section{Blood pressure variability in CFS}

The mechanical effect of ventilation is a significant source of blood pressure variability in the HF range (Malpas 2002; Zhang et al. 2002). Thus, our findings of reduced HF variability of SBP, which has not been previously reported, suggest either an altered ventilatory pattern or an altered effect of respiratory activity on SBP among CFS patients. There are several reports of reduced heart rate variability in the HF range, both at rest (Stewart 2000) and during orthostatic challenge in CFS patients (Stewart 2000; Wyller et al. 2007c, 2008a). Since respiratory activity is also the primary source of heart rate variability in the HF range (Saul 1990), the reduced HF variability of SBP observed here could be due to a reduced effect of respiration on heart rate (respiratory sinus arrhythmia). However, since respiratory patterns were not controlled and a quantitative respiratory signal was not measured in these studies, the precise origin of reduced SBP variability in the HF range cannot be determined. Future research could focus on possible alterations in CFS ventilatory patterns and the relationships between ventilation and both heart rate and blood pressure in these patients.

No differences between CFS patients and controls were observed for blood pressure variability in the LF range either at rest or during LBNP. An important source of such variability is sympathetically controlled vasomotion (Malpas 2002), which consequently appears to be unaltered in CFS. Although these findings are consistent with previously reported data from our laboratory (Wyller et al. 2007c), they contrast with Stewart's (2000) study of adolescent CFS patients, where increased blood pressure variability was noted in the LF range during $70^{\circ}$ head-up tilt. This apparent inconsistency could be entirely explained by different experimental protocols: First, LBNP and upright tilt, although similar in terms of physiological stress, do not result in identical hemodynamic responses. Second, LBNP of $-20 \mathrm{mmHg}$ used in this study, corresponding to head-up tilt of $20^{\circ}-30^{\circ}$, represents a much milder orthostatic challenge than $70^{\circ}$ head-up tilt and might have been insufficient to provoke a difference between the two groups.

\section{Dynamic closed-loop baroreflex assessment in CFS}

The baroreflex feedback mechanism includes both sympathetic and parasympathetic neural connections emanating from the brain stem cardiovascular control center to the sinus node (Lanfranchi and Somers 2002; Saul 1990). Because sympathetic heart rate control is relatively slow it only controls heart rate changes in the LF range, whereas the parasympathetic system has broad-band characteristics, operating in the LF as well as the HF range. Previous studies applying the same bivariate technique used here have demonstrated that during upright posture the $\alpha$-gain decreases in both LF and HF ranges (Barbieri et al. 2002), presumably secondary to parasympathetic withdrawal. With the milder orthostatic stress in this study, we observed a similar decrease for both controls and CFS patients but only in the HF range. Among CFS patients, $\alpha$-gain LF actually increased during LBNP. The net result was a marked and significant increase in the ratio $\alpha$-gain $\mathrm{LF} / \alpha$-gain $\mathrm{HF}$, strongly suggesting that in addition to the reduced parasympathetic heart rate control, there was enhanced sympathetic heart rate control mediated by the baroreflex.

The observed increase in the sympathetic component of the baroreceptor feedback with even mild orthostatic stress in CFS patients indicates early sympathetic activation and may reflect diminished baroreflex reserve for more severe stressors. These changes further suggest that the baroreflex may have a diminished ability to buffer a variety of internal and external influences on arterial pressure, but particularly those related to upright activity and ambulation, in line with our previous report on the combined effect of orthostatic stress and isometric exercise in CFS patients (Wyller et al. 2008b).

The function of the baroreceptor reflex has been addressed in two previous CFS studies. In a group of adolescent CFS patients and controls, Stewart (2000) reported significantly lower $\alpha$-gain in the HF as well as the LF range among CFS patients, both during rest and during $70^{\circ}$ head-up tilt. In adult CFS patients, Peckerman et al. (2003) reported enhanced decline in baroreceptor sensitivity upon standing as compared to controls. However, the sequential method adopted in the latter study does not allow separate estimation of LF and HF gain, making it most sensitive for parasympathetic heart rate control. Differences from the current study might be explained by different inclusion criteria, experimental protocols and mathematical algorithms. Taken together, however, like this study, they indicate a sympathetic predominance of baroreflex heart rate control during orthostatic stress in CFS patients.

\section{Cardiovascular dysregulation in CFS}

In agreement with past reports, the findings from this study confirm that CFS patients have functional disturbances of the autonomic nervous system affecting cardiovascular regulation. The underlying mechanism for this disturbance has been disputed. 
One possibility would be that CFS patients suffer from absolute or relative hypovolemia, which has indeed been reported previously (Farquhar et al. 2002; Streeten 2001; Streeten et al. 2000; Hurwitz et al. 2009). However, detailed studies of baroreflex function with techniques similar to ours have revealed a reduced LF baroreflex gain at rest following blood donation (Triedman et al. 1993) and furosemide treatment (Iwasaki et al. 2000) and a further attenuation of LF baroreflex gain in hypovolemic individuals during orthostatic stress (Triedman et al. 1993). Moreover, an attenuation of LF baroreflex gain has been demonstrated during experimentally induced hypovolemia by LBNP (Barbieri et al. 2002). Contrasting these results, we found equal LF baroreflex gain among controls and CFS patients at rest and increased LF baroreflex gain in CFS patients during orthostatic stress, making hypovolemia an unlikely explanation.

A second possibility would be cardiovascular deconditioning due to physical inactivity in CFS patients (De Lorenzo et al. 1998). The deconditioning influence on cardiovascular control appears to be partly a consequence of concomitant hypovolemia (Iwasaki et al. 2000), adding to the relevance of the reasoning above. Furthermore, in adults, both sedentary and gravitational deconditionings seem to be associated with attenuated sympathetic responsiveness during orthostatic stress (Levine et al. 1991; Sun et al. 2003), and endurance training in sedentary individuals tends to increase LF baroreflex gain at rest (Iwasaki et al. 2003). In a previous study of healthy adolescents, the degree of aerobic fitness did not predict cardiac autonomic responses during head-up tilt (Brunetto et al. 2005). Finally, no one of the CFS patients in this study was permanently bedridden, and previous evidence suggests that intermittent exposure to gravity during a bed-rest period is sufficient to prevent gravitational deconditioning (Sun et al. 2003; Zhang et al. 2000). Taken together, cardiovascular deconditioning does not seem to explain the results reported in this study.

A third possible explanation is a discrete disturbance of CNS autonomic control, such as temporary central resetting, redefining the homeostatic range of the baroreflex (Goldstein 2001). The smaller negative value for $\alpha$-phase LF among CFS patients as compared to controls during LBNP suggests a shortened response time within the sympathetic part of the baroreflex, possibly caused by central enhancement of neural transmission. Furthermore, as orthostatic challenge neither altered feedforward $\beta$-gain nor DBP variability in the LF range differently among CFS patients, the sympathetic predominance does not seem to be a reflection of blood pressure buffering, further pointing towards a central mechanism. Central resetting is not a common feature of orthostatic challenge, but does occur during aerobic exercise, resulting in attenuation of parasympathetic and predominance of sympathetic heart rate control (Macor et al. 1996; Spadacini et al. 2006), analogous to what we report in this study.

Data quality and study limitations

The responses among healthy adolescents to LBNP were similar in all respect to responses among healthy adults in previously reported LBNP experiments from our institutions, supporting the validity of this study (Barbieri et al. 2002). Furthermore, coherence values were well above 0.5 for all computed bivariate spectra, supporting the validity of the calculated gain and phase values.

Blood and/or plasma volume were not measured, leaving the question of hypovolemia unresolved. Finally, respiratory activity has been shown to change during orthostatic challenge and could therefore influence cardiovascular variability (Cooke et al. 1999); however, ventilation was not controlled for in this study.

\section{Concluding remarks}

Our findings of cardiovascular dysregulation point towards a discrete disturbance of CNS autonomic control in CFS patients. This may represent a distinct pathophysiologic phenomenon possibly conceptualized as a mismatch phenomenon, in which sensory input during orthostatic challenge evokes a disproportional or 'untuned' autonomic response. This concept is compliant with recently proposed theories of CFS pathophysiology, such as the theory of central sensitization (Yunus 2007) and our recently proposed theory of sustained arousal (Wyller et al. 2009). On a more general level, such a concept is in line with recent models on the mechanistic link between psychosocial stressors and cardiovascular morbidity (Goldstein 2001; Lucini et al. 2008). Further research should aim at exploring these relations in more detail, both in CFS and other related disorders.

Acknowledgments We thank Torun Flateb $\varnothing$, Department of Physiology, University of Oslo, Norway, for technical assistance during the experiments. Vegard Bruun Wyller has received a research grant from the University of Oslo.

Conflict of interest There are no conflicts of interests.

Open Access This article is distributed under the terms of the Creative Commons Attribution Noncommercial License which permits any noncommercial use, distribution, and reproduction in any medium, provided the original author(s) and source are credited.

\section{References}

Barbieri R, Waldmann RA, Di Virgilio V, Triedman JK, Bianchi A, Cerutti S, Saul JP (1996) Continuous quantification of baroreflex 
and respiratory control of heart rate by use of bivariate autoregressive techniques. Ann Noninvasive Electrocardiol 1:264-277

Barbieri R, Parati G, Saul JP (2001) Closed- vs open-loop assessment of heart rate baroreflex: implications for normal and abnormal blood pressure regulation and noninvasive assessment. IEEE Eng Med Biol Mag 20:33-42

Barbieri R, Triedman KJ, Saul JP (2002) Heart rate control and mechanical cardiopulmonary coupling to assess central volume: a systems analyses. Am J Physiol Regul Integr Comp Physiol 283:R1210-R1220

Boneva RS, Decker MJ, Maloney EM, Lin JM, Jones JF, Helgason DB, Reeves WC (2007) Higher heart rate and reduced heart rate variability persist during sleep in chronic fatigue syndrome: a population-based study. Auton Neurosci 137:94-101

Bou-Holaigah I, Rowe PC, Kan JS, Calkins H (1995) The relationship between neurally mediated hypotension and the chronic fatigue syndrome. JAMA 274:961-967

Brunetto AF, Roseguini BT, Silva BM, Hirai DM, Guedes DP (2005) Effects of gender and aerobic fitness on cardiac autonomic responses to head-up tilt in healthy adolescents. Pediatr Cardiol $26: 418-424$

Cho HJ, Skowera A, Cleare A, Wessely S (2006) Chronic fatigue syndrome: an update focusing on phenomenology and pathophysiology. Curr Opin Psychiatry 19:67-73

Cooke WH, Hoag JB, Crossman AA, Kuusela TA, Tahvanainen KU, Eckberg DL (1999) Human responses to upright tilt: a window on central autonomic integration. J Physiol 517:617-628

De Jong-De Vos van Steenwijk CC, Wieling W, Johannes JM, Harms MP, Kuis W, Wesseling KH (1995) Incidence and hemodynamic characteristics of near-fainting in healthy 6- to 16-year old subjects. J Am Coll Cardiol 25:1615-1621

De Lorenzo F, Xiao H, Mukherjee M, Harcup J, Suleiman S, Kadziola Z, Kakkar VV (1998) Chronic fatigue syndrome: physical and cardiovascular deconditioning. QJM 91:475-481

Duprez DA, DeBuyzere ML, Drieghe B, Vanhaverbeke F, Tas Y, Michielsen W, Clement DL (1998) Long- and short-term blood pressure and RR-interval variability and psychosomatic distress in chronic fatigue syndrome. Clin Sci 94:57-63

Farmer A, Fowler T, Scourfield J, Thapar A (2004) Prevalence of chronic disabling fatigue in children and adolescents. $\mathrm{Br} \mathrm{J}$ Psychiatry 184:477-481

Farquhar WB, Hunt BE, Taylor JA, Darling SE, Freeman R (2002) Blood volume and its relation to peak $\mathrm{O}(2)$ consumption and physical activity in patients with chronic fatigue. Am J Physiol Heart Circ Physiol 282:H66-H71

Franklin A (1998) How I manage chronic fatigue syndrome. Arch Dis Child 79:375-378

Fukuda K, Straus SE, Hickie I, Sharpe M, Dobbins JG, Komaroff A (1994) The chronic fatigue syndrome: a comprehensive approach to its definition and study. Ann Intern Med 121:953-959

Galland BC, Jackson PM, Savers RM, Taylor BJ (2008) A matched case control study of orthostatic intolerance in children/adolescents with chronic fatigue syndrome. Pediatr Res 63:196-202

Goldstein DS (2001) The autonomic nervous system in health and disease. Marcel Dekker, New York

Hisdal J, Toska K, Walloe L (2003) Design of a chamber for lower body negative pressure with controlled onset rate. Aviat Space Environ Med 74:874-878

Hurwitz BE, Coryell VT, Parker M, Martin P, Laperriere A, Klimas NG, Sfakianakis GN, Bilsker MS (2009) Chronic fatigue syndrome: illness severity, sedentary lifestyle, blood volume and evidence of diminished cardiac function. Clin Sci 118:125-135

Iwasaki K, Zhang R, Zuckerman JH, Pawelczyk JA, Levine BD (2000) Effect of head-down-tilt bed rest and hypovolemia on dynamic regulation of heart rate and blood pressure. Am J Physiol Regul Integr Comp Physiol 279:R2189-R2199

Iwasaki K, Zhang R, Zuckerman JH, Levine BD (2003) Doseresponse relationship of the cardiovascular adaption to endurance training in healthy adults: how much training for what benefit? J Appl Physiol 95:1575-1583

Lanfranchi PA, Somers VK (2002) Arterial baroreflex function and cardiovascular variability: interactions and implications. Am J Physiol Regul Integr Comp Physiol 283:R815-R826

Levine BD, Buckey JC, Fritsch JM, Yancy CW Jr, Watenpaugh DE, Snell PG, Lane LD, Eckberg DL, Blomqvist CG (1991) Physical fitness and cardiovascular regulation: mechanism of orthostatic intolerance. J Appl Physiol 70:112-122

Lucini D, Cannone V, Malacarne M, Bruno D, Beltrami S, Pizzinelli P, Piazza E, DiFede G, Pagani M (2008) Evidence of autonomic dysregulation in otherwise healthy cancer caregivers: a possible link with health hazards. Eur J Cancer 44:2437-2443

Macor F, Fagard R, Amery A (1996) Power spectral analyses of RR interval and blood pressure short-term variability at rest and during dynamic exercise: comparison between cyclists and controls. Int J Sports Med 17:175-181

Malpas S (2002) Neural influences on cardiovascular variability: possibilities and pitfalls. Am J Physiol Heart Circ Physiol 282:H6-H20

McCully KK, Smith S, Rajaei S, Leigh JS Jr, Natelson BH (2004) Muscle metabolism with blood restriction in chronic fatigue syndrome. J Appl Physiol 96:871-878

Natelson BH (2001) Chronic fatigue syndrome. JAMA 285:2557-2559

Parati G, Casadei R, Groppelli A, di Rienzo M, Mancia G (1989) Comparison of finger and intra-arterial blood pressure monitoring at rest and during laboratory testing. Hypertension 13:647-655

Peckerman A, LaManca JJ, Qureishi B, Dahl KA, Golfetti R, Yamamoto Y, Natelson BH (2003) Baroreceptor reflex and integrative stress responses in chronic fatigue syndrome. Psychosom Med 65:889-895

Rowe PC, Calkins H (1998) Neurally mediated hypotension and chronic fatigue syndrome. Am J Med 105:15S-21S

Rowe PC, Bou-Holaigah I, Kan JS, Calkins H (1995) Is neurally mediated hypotension an unrecognised cause of chronic fatigue? Lancet 345:623-624

Saul JP (1990) Beat-to-beat variations of heart rate reflect modulation of cardiac autonomic outflow. News Physiol Sci 5:32-37

Seifer CM, Kenny RA (2001) Head-up tilt testing in children. Eur Heart J 22:1968-1971

Spadacini G, Passino C, Leuzzi S, Valle F, Piepolo M, Calciati A, Sleight P, Bernardi L (2006) Frequency-dependent baroreflex control of blood pressure and heart rate during physical exercise. Int J Cardiol 107:171-179

Spence VA, Khan F, Belch JJ (2000) Enhanced sensitivity of the peripheral cholinergic vascular response in patients with chronic fatigue syndrome. Am J Med 108:736-739

Stevens PM, Lamb LE (1965) Effects of lower body negative pressure on the cardiovascular system. Am J Cardiol 16:506-515

Stewart J (2000) Autonomic nervous dysfunction in adolescents with postural orthostatic tachycardia syndrome and chronic fatigue syndrome is characterized by attenuated vagal baroreflex and potentiated sympathetic vasomotion. Pediatr Res 48:218-226

Streeten DH (2001) Role of impaired lower-limb venous innervation in the pathogenesis of the chronic fatigue syndrome. Am J Med Sci 321:163-167

Streeten DH, Thomas D, Bell DS (2000) The roles of orthostatic hypotension, orthostatic tachycardia, and subnormal erythrocyte volume in the pathogenesis of the chronic fatigue syndrome. Am J Med Sci 320:1-8 
Sun XQ, Yao YJ, Yang CB, Jiang SZ, Liang WB (2003) Effect of lower body negative pressure on orthostatic tolerance and cardiac function during 21 days head-down tilt bed rest. J Gravit Physiol 10:11-17

Tanaka H, Matsushima R, Tamai H, Kajimoto Y (2002) Impaired postural hemodynamics in young patients with chronic fatigue with and without orthostatic intolerance. J Pediatr 140:412-417

Triedman JK, Cohen RJ, Saul JP (1993) Mild hypovolemic stress alters autonomic modulation of heart rate. Hypertension 21:236-247

Wyller VB, Due R, Saul JP, Amlie JP, Thaulow E (2007a) Usefulness of an abnormal cardiovascular response during low-grade headup tilt-test for discriminating adolescents with chronic fatigue from healthy controls. Am J Cardiol 99:997-1001

Wyller VB, Godang K, Mørkrid L, Saul JP, Thaulow E, Walløe L (2007b) Abnormal thermoregulatory responses in adolescents with chronic fatigue syndrome: relation to clinical symptoms. Pediatrics 120:e129-e137

Wyller VB, Saul JP, Amlie JP, Thaulow E (2007c) Sympathetic predominance of cardiovascular regulation during mild orthostatic stress in adolescents with chronic fatigue. Clin Physiol Funct Imaging 26:1-8

Wyller VB, Barbieri R, Thaulow E, Saul JP (2008a) Enhanced vagal withdrawal during mild orthostatic stress in adolescents with chronic fatigue. Ann Noninvasive Electrocardiol 13:67-73
Wyller VB, Saul JP, Walløe L, Thaulow E (2008b) Enhanced sympathetic response during orthostatic stress and attenuated sympathetic responses during isometric exercise may account for clinical symptoms in adolescents with chronic fatigue. Eur $\mathbf{J}$ Appl Physiol 102:623-632

Wyller VB, Malterud K, Eriksen HR (2009) Can sustained arousal explain Chronic Fatigue Syndrome? Behav Brain Funct 5:10

Yataco A, Talo H, Rowe P, Kass DA, Berger RD, Calkins H (1997) Comparison of heart rate variability in patients with chronic fatigue syndrome and controls. Clin Auton Res 7:293-297

Yoshiuchi K, Quigley KS, Ohashi K, Yamamoto Y, Natelson BH (2004) Use of time-frequency analysis to investigate temporal patterns of cardiac autonomic response during head-up tilt in chronic fatigue. Auton Neurosci 113:55-62

Yunus MB (2007) Fibromyalgia and overlapping disorders: the unifying concept of central sensitivity syndromes. Semin Arthritis Rheum 36:339-356

Zhang LN, Gao F, Ma J, Zhang LF (2000) Daily head-up tilt, standing or centrifugation can prevent vasoreactivity changes in arteries of simulated weightless rats. J Gravit Physiol 7:P143-P144

Zhang R, Iwasaki K, Zuckerman JH, Behbehani K, Crandall CG, Levine BD (2002) Mechanism of blood pressure and RRvariability: insights from ganglion blockade in humans. J Physiol 543:337-348 